\title{
Is exclusion of leukocytes from platelet-rich plasma (PRP) a better choice for early intervertebral disc regeneration?
}

\author{
Shan-zheng Wang ${ }^{1,2}$, Wei-min Fan ${ }^{1 *}$, Jun Jia ${ }^{2}$, Liang-yu $\mathrm{Ma}^{2}$, Jia-bin $\mathrm{Yu}^{2}$ and Chen Wang ${ }^{2^{*}}$
}

\begin{abstract}
Background: Platelet-rich plasma (PRP) is becoming a promising strategy to treat early intervertebral disc degeneration (IDD) in clinics. Pure PRP without leukocytes (P-PRP) may decrease the catabolic and inflammatory changes in the early degenerated intervertebral discs. The aim of this study was to investigate the effects of P-PRP on nucleus pulposus-derived stem cells (NPSCS) isolated from early degenerated intervertebral discs in vitro.

Methods: NPSCs isolated from early degenerated discs of rabbits were treated with P-PRP or leukocyte-platelet-rich PRP (L-PRP) in vitro, followed by measuring cell proliferation, stem cell marker expression, inflammatory gene expression, and anabolic and catabolic protein expression by immunostaining, quantitative real-time polymerase chain reaction, Western blot, and enzyme-linked immunosorbent assay.

Results: Cell proliferation was induced by P-PRP in a dose-dependent manner with maximum proliferation at 10\% P-PRP dose. P-PRP induced differentiation of NPSCs into active nucleus pulposus cells. P-PRP mainly increased the expression of anabolic genes and relative proteins, aggrecan (AGC), collagen types II (Col II), while L-PRP predominantly increased the expression of catabolic and inflammatory genes, matrix metalloproteinase-1 (MMP-1), MMP-13, interleukin-1 beta (IL-1 $\beta$ ), IL-6, tumor necrosis factor alpha (TNF- $a$ ), and protein production of IL-1 $\beta$ and TNF- $a$.

Conclusions: Leukocytes in PRP activate inflammatory and catabolic effects on NPSCs from early degenerated intervertebral discs. Hence, P-PRP may be a more suitable therapeutic strategy for early IDD.
\end{abstract}

Keywords: Platelet-rich plasma, Leukocyte- and platelet-rich plasma, Pure platelet-rich plasma, Intervertebral disc degeneration, Nucleus pulposus, Stem cells

\section{Background}

As a major cause of low back pain, intervertebral disc degeneration (IDD) is a serious clinical problem, causing enormous financial and health-care costs $[1,2]$. Conservative treatments, including nonsteroidal anti-inflammatory drugs and physiotherapy, can often alleviate the symptoms but without prohibiting the degenerative process. Spinal surgeries are extensively accepted as an effective strategy to treat IDD with worsening neurological symptoms [3, 4]. However, after removing the herniated discs or even fusing the adjacent spinal segments, the neighboring discs

\footnotetext{
*Correspondence: fanweimin2017@163.com; wangchen_seu_edu@163.com ${ }^{1}$ The First Affiliated Hospital of Nanjing Medical University, 300 Guangzhou Road, Nanjing, Jiangsu 210029, People's Republic of China

${ }^{2}$ Department of Orthopaedics, Zhongda Hospital, Medical School of Southeast University, 87 Ding Jia Qiao Road, Nanjing, Jiangsu 210009, People's Republic of China
}

may experience an increasing progression of degeneration and instability [5]. In recent years, as a choice of biological strategies, platelet-rich plasma (PRP) is drawing increasing attention both in clinical treatment and basic scientific research [6-8].

PRP is defined as a volume of the autologous plasma with high platelet concentration above baseline $[9,10]$. The concept that PRP treatment can reverse the progression of IDD is based on the role of platelets in wound-healing [10-14]. When activated, a variety of growth factors, including platelet-derived growth factor (PDGF), transforming growth factor- $\beta$ (TGF- $\beta$ ), vascular endothelial growth factor-A (VEGF-A), basic fibroblast growth factor (bFGF), epidermal growth factor (EGF), and connective tissue growth factor (CTGF), among others, are secreted from platelets $[15,16]$. All these growth factors play a joint role when applied in 
intervertebral disc regeneration. Despite the wide use of PRP, there are no standardized protocols in PRP preparation, resulting in variations in componential composition, in particular, leukocytes [17]. It has been demonstrated that leukocytes in PRP can release high levels of pro-inflammatory cytokines, such as IL- $1 \beta$ and TNF- $\alpha$, which increase the catabolism of extracellular matrix (ECM) $[18,19]$. However, leukocytes in PRP can be beneficial to stimulate the immune response against infection and promote chemotaxis, proliferation, and differentiation of cells [20]. Therefore, it is necessary to define the precise effects of PRP on degenerated discs so that its clinical applications can be more accurate and beneficial.

Leukocyte-platelet-rich PRP (L-PRP) is often used to treat open traumatic injuries [21]. However, high levels of pro-inflammatory cytokines in L-PRP may activate the catabolic and inflammatory changes to counter the beneficial effect of the growth factors on tissue regeneration. Considering the detrimental effects of leukocytes on tissue regeneration, to exclude the leukocytes in PRP for pure PRP (P-PRP) is an attempt to achieve maximum therapeutic potential.

As a core component of intervertebral disc, nucleus pulposus (NP) is playing an important role in the mechanical and biological homeostasis of intervertebral discs [22]. In recent years, NP -derived stem cells (NPSCs) were isolated and characterized [23, 24]. As a key role in intervertebral disc homeostasis, these endogenic stem cells self-renew and differentiate into NP cells that are responsible for the self-regeneration of the NP when activated. While the superior effects of P-PRP over L-PRP have been proved on tendon [25], bone [26] and cartilage [27] healing, they have not yet been elucidated on intervertebral disc regeneration. Also, how P-PRP differentiate from L-PRP in influencing the endogenic NPSCs when repairing the early degenerated intervertebral discs is largely unknown. Therefore, we designed this study to determine effects of P-PRP on NPSCs isolated from early degenerated intervertebral discs.

\section{Methods}

\section{Preparation and quantification of P-PRP and L-PRP}

The Institutional Animal Care and Use Committee of Southeast University approved the protocol for the use of rabbits in this study. In total, 20 adult New Zealand White rabbits (6-8 months old, $3.0-4.0 \mathrm{~kg}$ ) were used in this study, and were randomly used in either P-PRP group or L-PRP group ( $n=10$ each). P-PRP and L-PRP were prepared according to a two-step centrifugation method as previously described [27]. Briefly, the fresh whole blood was collected into acid citrate dextrose solution A (ACD-A) anticoagulant $(1 \mathrm{~mL}$ ACD-A/9 mL blood) and centrifuged at $160 \mathrm{~g}$ for $10 \mathrm{~min}$. After the first centrifugation, the blood was separated into three layers: erythrocytes at the bottom, buffy coat in the middle, and platelet-containing plasma at the top. Then, the top layer was transferred to a new tube and spun again at $1000 \mathrm{~g}$ for $10 \mathrm{~min}$. After discarding the supernatant (platelet-poor plasma), the remaining plasma and precipitated platelets were blended evenly to obtain $1 \mathrm{~mL}$ of P-PRP. L-PRP was prepared based using a buffy coat two-step centrifugation method. In brief, a centrifugation at $250 \mathrm{~g}$ for $10 \mathrm{~min}$ was used to separate three layers as above. Then, the top two layers were collected and further centrifuged at $1000 \mathrm{~g}$ for $10 \mathrm{~min}$. After the second centrifugation, the supernatant (platelet-poor plasma) was discarded, and the precipitated platelets were resuspended in the remaining $1 \mathrm{~mL}$ of plasma to obtain L-PRP. Leukocyte and platelet concentrations in L-PRP, P-PRP, and whole blood were measured by an automatic hematology analyzer (XE-2100, Sysmex, Kobe, Japan).

\section{Isolation of rabbit NPSCs from early degenerated and healthy discs}

The rabbit lumbar IDD models were established as previously described [28]. After 2 weeks of intervention, the early degenerated disc model was evaluated by a $3.0 \mathrm{~T}$ magnetic resonance imaging (MRI). The NP tissues of the early degenerated discs and healthy discs (control group) were isolated and cultured. Briefly, the harvested $\mathrm{NP}$ tissues were minced and digested using $0.2 \mathrm{mg} / \mathrm{ml}$ collagenase II (Sigma-Aldrich, St. Louis, Mo, USA) in Dulbecco's modified Eagle's medium with low glucose (DMEM-LG, Gibco, Waltham, MA, USA) for 4-6 h. The suspension was then centrifuged at $1000 \mathrm{rpm}$ for $10 \mathrm{~min}$. The cell pellet was re-suspended in DMEM-LG supplemented with $10 \% \mathrm{FBS}, 100 \mathrm{U} / \mathrm{mL}$ penicillin, $100 \mathrm{mg} / \mathrm{mL}$ streptomycin. The cells were diluted to 10 cells/uL and seeded into six-well plates in a humidified incubator at $37{ }^{\circ} \mathrm{C}$ with $5 \% \mathrm{CO}_{2}$. After 15 days, NPSCs colonies observed in the wells were trypsinized for further culture. NPSCs at passage 2 were used for further experiments. To compare the formed colonies, the initially plated cells from NP tissues were cultured for 10 days, washed three times by PBS, fixed with $10 \%$ methanol for 15 min and stained with $0.5 \%$ crystal violet (Sigma-Aldrich) for $15 \mathrm{~min}$ for further observation.

\section{Polymerase chain reaction (PCR) for stem cell markers}

Total RNA was extracted from cells and then reverse transcribed to cDNA using a reverse transcription kit (Fermentas, Waltham, MA, USA) after DNase I treatment (Fermentas). The genes included MSC positive marker genes (CD29, CD44, CD166), MSC negative marker genes (CD4, CD8, CD14), and housekeeping gene GAPDH [29]. All the primer sequences are listed in Table 1. The sequences were designed according to published papers or designed by ourselves. All the primers were synthesized by Invitrogen. 
Table 1 Primers used in polymerase chain reaction and quantitative real-time polymerase chain reaction for gene expression analysis

\begin{tabular}{|c|c|}
\hline Gene & Primer sequence ( $5^{\prime}$ to $\left.3^{\prime}\right)$ \\
\hline CD29-F & GTCACCAACCGTAGCAA \\
\hline CD29-R & СTCСTCATCTCATTCATCAG \\
\hline CD44-F & CGATTTGAATATAACCTGCCGC \\
\hline CD44-R & CGTGCCCTTCTATGAACCCA \\
\hline CD166-F & GGACAGCCCGAAGGAATACGAA \\
\hline CD166-R & GACACAGGCAGGGAATCACCAA \\
\hline CD4-F & GATGGAGGTGGAACTGC \\
\hline CD4-R & GGAAAGCCCAACACTATG \\
\hline CD8-F & GGGTGGAAAAGGAGAAGC \\
\hline CD8-R & AGGTGAGTGCGGGAGAC \\
\hline CD14-F & CAGGTGCCTAAGGGACT \\
\hline CD14-R & AATAAAGTGGGAAGCGG \\
\hline Coll II-F & CAGGATGTCCAGGAGGCT \\
\hline Coll II-R & GCAGTGGCGAGGTCAGTAG \\
\hline AGC-F & GGAGCCCGAGCCTATACTATTT \\
\hline AGC-R & CCCAAGGACCACCAATCA \\
\hline IL-6-F & AGGGAGGTCGAGCTGTTCTC \\
\hline IL-6-R & GGAGTGTTCACTAAGCGGTCA \\
\hline $\mid L-1 \beta-F$ & CGGTCAAGGAGAGGAGCTTAC \\
\hline$I L-1 \beta-R$ & GGACTAGCCCTCGCTTATCTIT \\
\hline TNF-a-F & GGAGAAGCCGGTAGTGGAGAT \\
\hline TNF-a-R & GGTCTGGTCACGGTTTGGAA \\
\hline IL-8-F & CACAGTGGACGACATCCGAAA \\
\hline IL-8-R & AGCTACATAGGAATTACGGGCAA \\
\hline MMP-1-F & CGACTCGCTATCTCCAAGTGA \\
\hline MMP-1-R & GTTGAACCAGTCTCCGACCA \\
\hline MMP-13-F & GGAGGCGAGAACATCAAGCC \\
\hline MMP-13-R & CGGCCTTCCCTCGTAGTGA \\
\hline OCT4-F & ACCTTCATCGGAAACTCCAAAG \\
\hline OCT4-R & ACTGTTAGGCTCAGGTGAACT \\
\hline Nanog-F & CTGTGGGTTTCTGTGCTGG \\
\hline Nanog-R & CCGGCTTCAAGGCTTTCAG \\
\hline GAPDH-F & ACTTTGTGAAGCTCATTTCCTGGTA \\
\hline GAPDH-R & GTGGTTTGAGGGCTCTTACTCCTT \\
\hline
\end{tabular}

\section{Induced differentiation potential of isolated NPSCs}

The induced differentiation methods were described previously [30, 31]. Briefly, passage 2 NPSCs were seeded at a density of $4 \times 10^{4}$ cells/well in a 24 -well plate in basic culture medium (DMEM-LG) supplemented with $10 \%$ FBS, $100 \mathrm{U} / \mathrm{ml}$ penicillin, $100 \mathrm{mg} / \mathrm{ml}$ streptomycin. They were subjected to induced differentiation by culturing them in osteogenic (Cyagen Biosciences, Santa Clara, CA, USA, RASMX-90021), adipogenic (Cyagen Biosciences,
RASMX-90031) and chondrogenic (Cyagen Biosciences, RASMX-90041) medium, respectively. The outcomes were evaluated using Alizarin Red, Oil Red O and Alcian Blue staining, respectively.

\section{NPSCs proliferation assay}

NPSCs were seeded at the density of $1 \times 10^{4}$ in a 24-transwell system and cultured in DMEM-LG containing P-PRP or L-PRP at different concentrations: $0 \%, 5 \%$, $10 \%, 15 \%$, and $20 \%$ (vol/vol). Cell growth was tested on day 3 and 7 by Cell Counting Kit-8 (CCK-8) (Sigma-Aldrich). Fresh culture medium containing $10 \%$ CCK- 8 solution was added and incubated at $37{ }^{\circ} \mathrm{C}$ for $2.5 \mathrm{~h}$. Then, the absorbance was measured by a microplate reader (SPECTRA max Plus 384, Molecular Devices, San Jose, CA, USA) at $450 \mathrm{~nm}$. Each treatment was replicated three times and the absorption values were independently calculated as OD 450 $\mathrm{nm}_{\text {experiment }}$ - OD 450nm blank. The average absorption value of all replicates represented cell proliferation in each group.

\section{Induced cell morphology}

NPSCs were seeded in a 24-transwell system at a density of $1 \times 10^{4}$ per well and incubated in growth medium (DMEM-LG + 10\% FBS) alone (control group) or growth medium with $10 \%$ P-PRP (P-PRP group) or $10 \%$ L-PRP (L-PRP group). The culture medium was changed every 3 days. After 14 days, cell morphology was observed under the inverted microscope.

\section{Western blot analysis}

The Western blot analysis was described previously [30, 31]. The cell culture medium was changed every 3 days in three groups as stated above. After 14 days, NPSCs were harvested and centrifuged to obtain cells in each group. Total proteins were then isolated by a mammalian protein extraction reagent (M-PER) (Thermo Fisher Scientific, Waltham, MA, USA, catalog no.78505) containing 1.5\% protease inhibitors (Sigma-Aldrich) and then centrifuged at $14,000 \mathrm{~g}$ for $10 \mathrm{~min}$. The supernatant was stored at $4{ }^{\circ} \mathrm{C}$. Protein concentration of the supernatant was evaluated by the BCA protein Assay Reagent Kit (Pierce, Waltham, MA, USA). Equal amounts of total protein from each group were then separated on 12\% SDS-PAGE gels (Thermo Fisher Scientific) at a constant $100 \mathrm{~V}$ for $60 \mathrm{~min}$. Proteins were transferred to a PVDF membrane (EMD Millipore, Burlington, MA, USA) at $100 \mathrm{~V}$ for $30 \mathrm{~min}$. The membrane was then blocked in 5\% dry milk/TBS-Tween 20 for $1 \mathrm{~h}$ at room temperature and then incubated overnight with anti-collagen type II (Col II) antibody (arigo Bio, Hsinchu City, Taiwan, ROC), anti-Agrecan antibody (Novus Biologicals, Littleton, CO, USA) at a dilution of 1:1000. The membranes were washed in PBS/Tween-20 three times for $10 \mathrm{~min}$ each and further incubated with 
peroxidase-conjugated goat anti-mouse antibody (Santa Cruz Biotechnology, Dallas, TX, USA) at a dilution of 1:5000 in 1\% dry milk/PBS for $1 \mathrm{~h}$ at room temperature. Finally, the protein bands were detected by an enhanced chemiluminescence (ECL) detection kit (Amersham Biosciences, Piscataway, NJ, USA), followed by exposing the membrane to X-ray films for visualization. Mouse antihuman GAPDH (Chemicon, Temecula, CA, USA) was used as an internal control to verify the loading of equal amounts of proteins in each well.

\section{Gene expression analysis using quantitative real-time polymerase chain reaction (qRT-PCR)}

To determine the effects of P-PRP and L-PRP on the gene expression of NPSCs, quantitative real-time polymerase chain reaction (qRT-PCR) was used to analyze the following genes as previously described [30, 31]: stem cell-related gene (Oct-4, Nango), NP-related genes (Col II and AGC), catabolic genes (MMP-1, MMP-13), and inflammatory marker genes (IL-1 $\beta$, IL- 6 , IL- 8 , and TNF- $\alpha$ ). The sequences of primers used in the reactions are listed in Table 1. Each reaction had at least three replicates, and the relative expression of each target gene was calculated by using the $2^{-\Delta \Delta C T}$ formula.

\section{Measuring of IL-1 $\beta$ and TNF- $\alpha$}

First, NPSCs were cultured as above either in growth medium alone (control) or in growth medium containing 10\% P-PRP or 10\% L-PRP. At least three replicates were maintained for each group. After 4 days, cells were obtained by trypsin. The cell pellet was used to estimate the cell count by an auto cellometer (Cellometer Auto T4; Nexcelom Bioscience LLC, Lawrence, MA, USA), and the supernatant was used to measure interleukin (IL)-1 $\beta$ and tumor necrosis factor (TNF)- $\alpha$ levels by a specific enzyme-linked immunosorbent assay (ELISA) kits according to the instructions of the manufacturer (Shanghai Westang Biotechnology, Shanghai, China).

\section{Immunostaining of induced NPSCs}

NPSCs cultured as above for 14 days were collected by trypsinization and fixed for $20 \mathrm{~min}$ in $4 \%$ paraformaldehyde/PBS. The collected cells were washed with $0.1 \%$ Triton-X100/PBS for $5 \mathrm{~min}$. Immunostaining for NP-related proteins, aggrecan and, Col II was performed by blocking the fixed cells in $2 \%$ mouse serum. Cells were then incubated with mouse monoclonal anti-AGC antibody (Novus Biologicals), anti-collagen II antibody (arigo Bio) overnight. After washing in PBS three times for 5 min each, the cells were further incubated with Cy3-conjugated goat anti-mouse IgG secondary antibody (1:500; EMD Millipore; catalog no. AP124X4-K) for $1.5 \mathrm{~h}$. After the final wash in PBS, the stained cells were examined by an inverted fluorescence microscope (Olympus, Tokyo, Japan, IX51).

\section{Statistical analysis}

Results are presented as mean \pm standard deviation (SD) with no less than three replicates for each experimental condition. Statistical analyses were performed using one-way analysis of variance (ANOVA) followed by Fisher's protected least significant difference for multiple comparisons. For statistical analysis of other results, two-tailed, paired, or unpaired Student's $t$ test was performed. Differences were considered significant when $P$ values were below 0.05 .

\section{Results}

\section{Characterization of L-PRP and P-PRP}

Similar platelet concentrations were observed in L-PRP $\left(1691.75 \pm 151.89 \times 10^{9} / \mathrm{L}\right)$ and P-PRP $(1749.13 \pm 128.35 \times$ $10^{9} / \mathrm{L}$ ), which were three times higher than the basic platelet level in the whole blood $\left(440.50 \pm 60.18 \times 10^{9} / \mathrm{L}\right)$ (Fig. 1a). The average leukocyte concentration in the whole blood was $8.92 \pm 1.03 \times 10^{9} / \mathrm{L}$. However, in L-PRP, leukocyte concentration was $22.97 \pm 2.63 \times 10^{9} / \mathrm{L}$, while this concentration was negligible in P-PRP $(0.23 \pm 0.09 \times$ $10^{9} /$ L) (Fig. 1b).

\section{Isolation and culture of NPSCs}

Compared to the healthy discs (Fig. 2a), the punctured discs exhibited decreasing signal intensity after 2 weeks of intervention (Fig. 2b). The worsening degenerative trend was observed at week 4 (Fig. 2c). The NP tissues (Fig. 2d) were collected from the early degenerated discs (2 weeks after intervention). The NP-derived cells isolated from early degenerated discs formed colonies after 10 days as indicated by crystal violet staining (Fig. 2e). The morphology of cells in the colonies also varied, with some of them being cobblestone-like and others being spindle-like (Fig. 2f). At P2, a homogeneous population of cobblestone-like cells was observed (Fig. 2g). As a control, NPSCs isolated from healthy rabbit discs formed more colonies (Fig. 2h), but shared similar morphology (Fig. 2i, j).

\section{Expression of stem cell markers in NP-derived colony- forming cells}

The gene expression of typical MSC-associated surface antigens was tested by PCR (Fig. 3). The NP-derived colony-forming cells isolated from early degenerated discs (D-NPSCs) had strong expression of CD29, CD44, and CD166, which are usually positive in MSCs. Meanwhile, they had negligible expression of CD4, CD8, and CD14, which seldom exist in MSCs. The expression of MSC markers was similarly positive in NPSCs isolated from healthy rabbit discs (H-NPSCs). As a positive control, rabbit spleen cells 


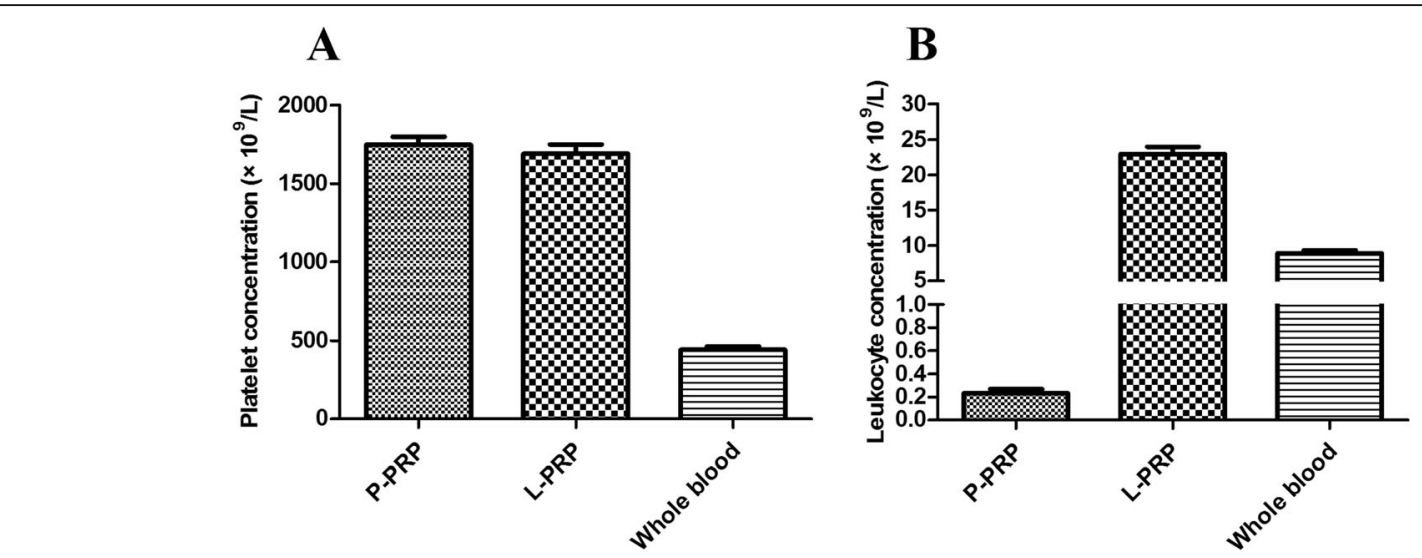

Fig. 1 Composition of P-PRP, L-PRP and whole blood. a Platelet concentration $\left(\times 10^{9} / \mathrm{L}\right)$. b Leukocyte concentration $\left(\times 10^{9} / \mathrm{L}\right)$. P-PRP pure platelet-rich plasma, L-PRP leukocyte-platelet-rich plasma

were found to express all the above genes. As a negative control, NP cells showed negligible expression of all the above genes.

\section{Multi-potential differentiation of NPSCs}

The rabbit NP-derived colony-forming cells from the early degenerated discs were subjected to induced differentiation processes (osteogenesis, adipogenesis, and chondrogenesis) to determine the multi-differentiation potential. After 3 weeks for osteogenic induction, calcium deposits were highly visible in the induced cells, which were fixed and stained with Alizarin Red (Fig. 4a). During adipogenic induction, the rabbit NPSCs started to secrete oil droplets, which were fixed and stained with Oil Red O staining (Fig. 4b). After 3 weeks for chondrogenic induction, active production of sulfated proteoglycans in the induced cells was validated by Alcian Blue staining (Fig. 4c).
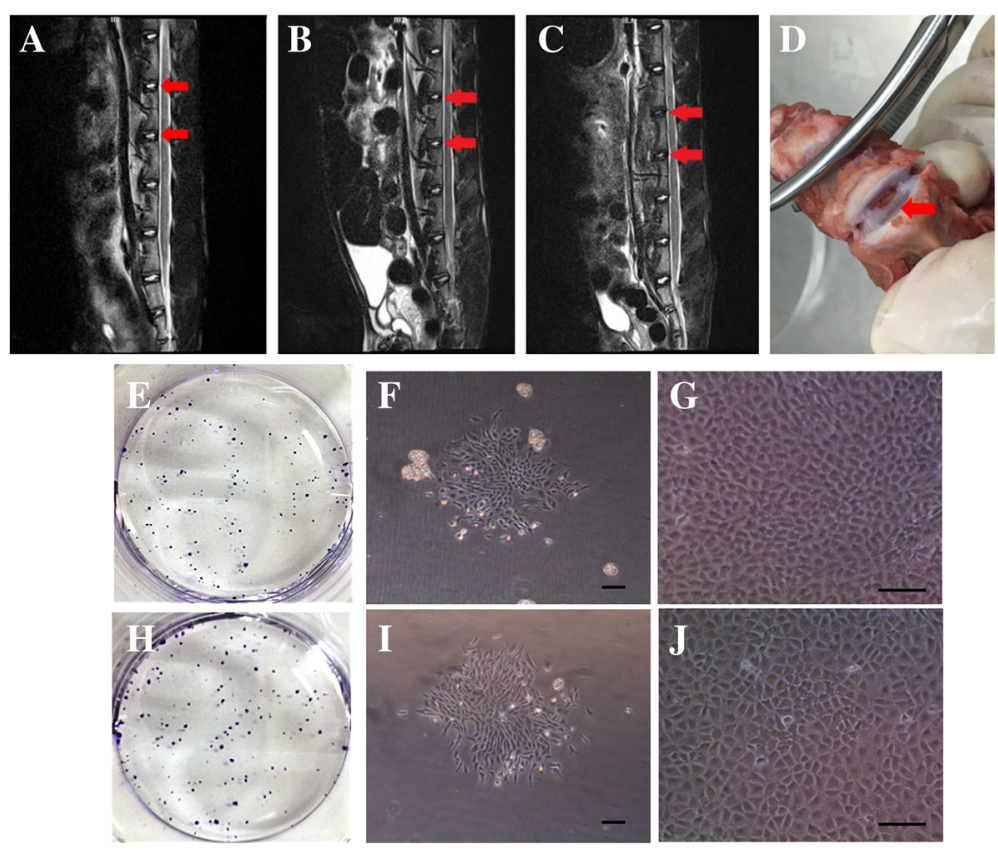

Fig. 2 Isolation and culture of NPSCs. The MRI T2-weighted signal of targeted discs (red arrows) was in high intensity in the heathy rabbit (a). The punctured discs (red arrows) showed decreasing signal intensity at week 2 (b), and this signal intensity worsened gradually at week 4 (b). The degenerated discs were obtained 2 weeks after intervention, and the NP tissue (red arrow) could be seen in the middle of the discs (d). NPSCS isolated from early degenerated discs formed colonies after in vitro culture for 10 days as indicated by crystal violet staining (e). The morphology of cells in the colonies varied, with some of them being cobblestone-like and others being spindle-like (f). As a control, cells isolated from healthy rabbit discs formed more colonies $(\mathbf{h})$, but shared similar morphology $(\mathbf{i}, \mathbf{j})$. Scale bars, $100 \mathrm{~mm}$. MRI magnetic resonance imaging, NP nucleus pulposus, NPSCS nucleus pulposus-derived stem cells 


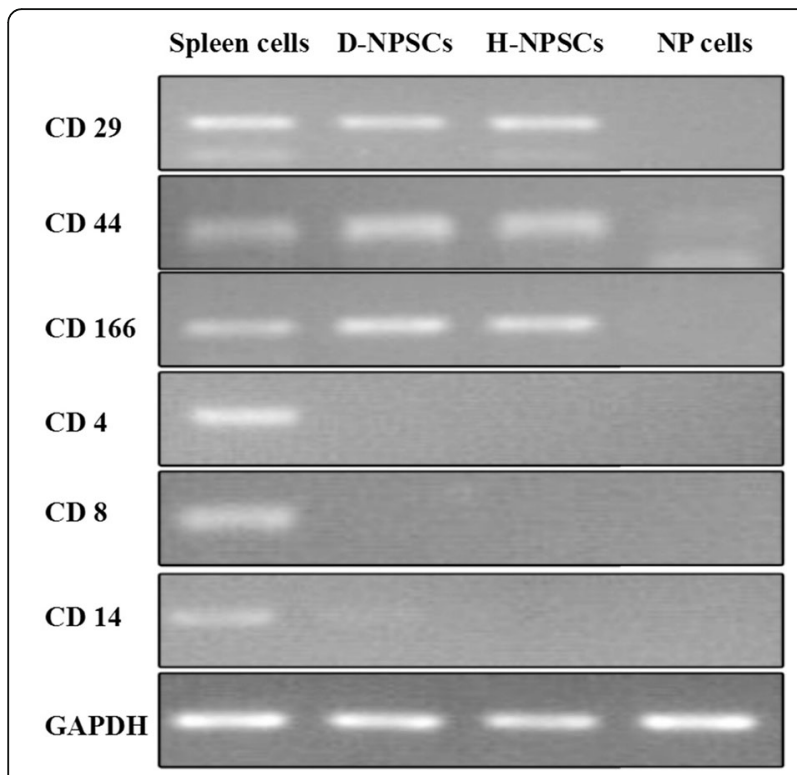

Fig. 3 Expression of stem cell markers in NP-derived colony-forming cells. NP-derived colony-forming cells both from the early degenerated discs (D-NPSCs) and healthy discs (H-NPSCs) were positive for CD29, CD44, and CD166, but negative for CD4, CD8 and CD14. Spleen cells expressed all these genes as a positive control, while NP cells expressed none of these genes. NP nucleus pulposus

\section{NPSCs proliferation rate is PRP dose-dependent}

In the presence of P-PRP or L-PRP, cell proliferation rate increased in a PRP dose-dependent manner (Fig. 5). However, increasing PRP dose over 15\% descended the proliferation trend. At each time point, 10\% PRP concentration induced significantly higher cell proliferation rate compared with other groups. Since maximum proliferation rate was induced by $10 \%$ PRP, this dose was used for further analyses.

\section{P-PRP and L-PRP specifically induces NPSCs into active NP cells}

NPSCs morphology in the controls maintained the shape of cobblestone-like cells (Fig. 6a). However, after the treatment of 10\% L-PRP or 10\% P-PRP, NPSCs proliferated faster and the shape changed from cobblestone-like to fibroblast-like shape (Fig. 6b, c). Two markers of active NP cells, including AGC and Col II increased significantly compared to the control group (Fig. 6d, e). P-PRP yielded the highest gene expression among the three groups. Furthermore, analysis of stem cell markers, including Nanog and OCT-4, indicated that both P-PRP and L-PRP decreased the stemness of NPSCs in vitro (Fig. 6f, g). Western blot analysis also validated these results (Fig. 6h). Immunostaining of the active NP cell protein, AGC, and Col II, was confirmed in high intensity after L-PRP or P-PRP treatment, while this was negligible in the control group. P-PRP treatment resulted in the highest protein production among the three groups (Fig. 6i).

\section{L-PRP induces higher levels of inflammatory responses in NPSCs from early degenerated discs}

To investigate the effects of L-PRP and P-PRP on the inflammatory responses, we first examined the expression levels of the inflammatory genes, IL-1 $\beta$, TNF- $\alpha$, IL- 6 , and IL-8, by qRT-PCR. Compared to the control group, P-PRP and L-PRP both decreased the expression of inflammatory genes (Fig. 7a-d). Also, we noticed that the inflammatory gene expression was significantly lower in the P-PRP group compared to that in the L-PRP group. Moreover, the levels of inflammatory cytokines (IL-1 $\beta$, TNF- $\alpha$ ) in the cell supernatants were consistent with the above gene expression data (Fig. 7e-f).

\section{P-PRP downregulates more extensive catabolic responses in NPSCs from early degenerated discs}

P-PRP and L-PRP both significantly downregulated the gene expression of MMPs (MMP-1, MMP-13) when compared with the control. P-PRP significantly downregulated the above catabolic genes compared with L-PRP (Fig. 8a, b).

\section{Discussion}

PRP therapy has been widely applied in clinical repair and regeneration of different tissues and organs [16, 32, 33]. Many human clinical trials and basic scientific researches support the use of PRP not only for its autologous origin, but also for its activation of the tissue repair
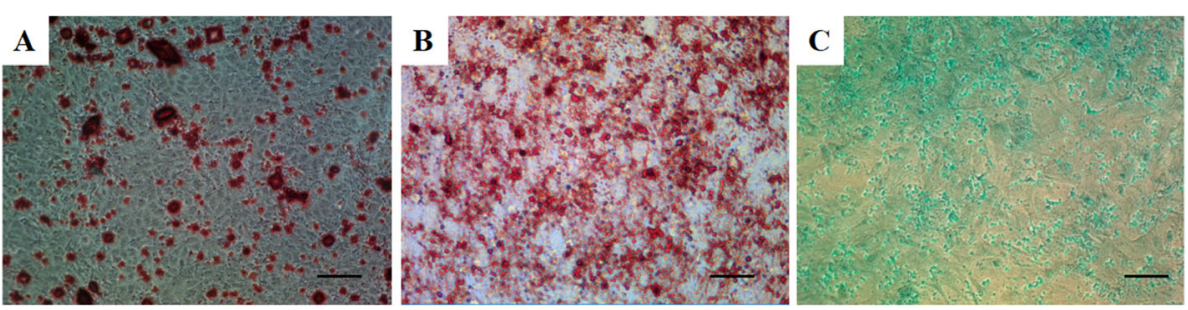

Fig. 4 Multi-potential differentiation of NPSCs. Osteogenic differentiation at 3 weeks. Mineralization was positive for Alizarin Red staining (a). Adipogenic differentiation at 2 weeks. Secretion of oil droplets were positive for Oil Red O staining (b). Chondrogenic differentiation at 3 weeks. Cells were stained with Alcian Blue staining (c). Scale bars, $100 \mathrm{~mm}$. L-PRP leukocyte-platelet-rich plasma, NPSCs nucleus pulposus-derived stem cells, P-PRP pure platelet-rich plasma 


\section{P-PRP}

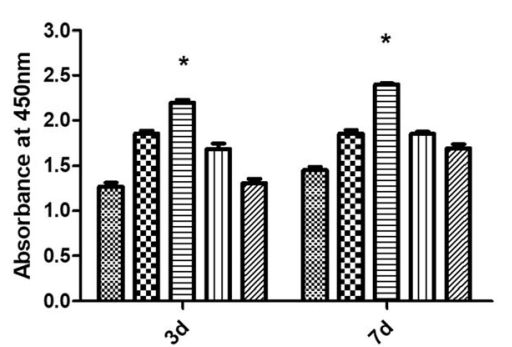

L-PRP

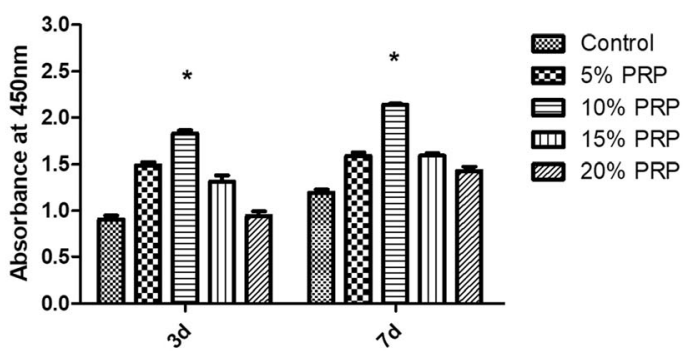

Fig. 5 Proliferation of NPSCs cultured in various concentrations of P-PRP or L-PRP. Cell proliferation was measured on day 3 and 7 in culture. Cells proliferated in a dose-dependent manner with 10\% P-PRP and 10\% L-PRP inducing the maximum effects at each time point. Asterisks indicate significant differences $(P<0.05)$ when compared with the controls. L-PRP leukocyte-platelet-rich plasma, NPSCs nucleus pulposus-derived stem cells, P-PRP pure platelet-rich plasma

process [34-36]. PRP was proved promising in IDD by valuable clinical trials and animal studies [6, 37, 38]. However, inconsistency in composition of PRP prepared by different methods may have contributed to different results [39]. Leukocytes were suggested for their pro-inflammatory effect in PRP and were also proposed to be excluded in bone defects, chronic tendon injury, and osteoarthritis treatment [25]. Therefore, in this study, we determined whether the exclusion of leukocytes in PRP (P-PRP) was more valuable in repair and regeneration of early IDD.

In recent years, the endogenic stem cells from the NP tissues were isolated and characterized for its potential in self-renewal and tissue regeneration [23, 24]. In light of this, we tried to isolate stem cells from rabbit NP tissues in the early degenerated stage, which may potentially be a

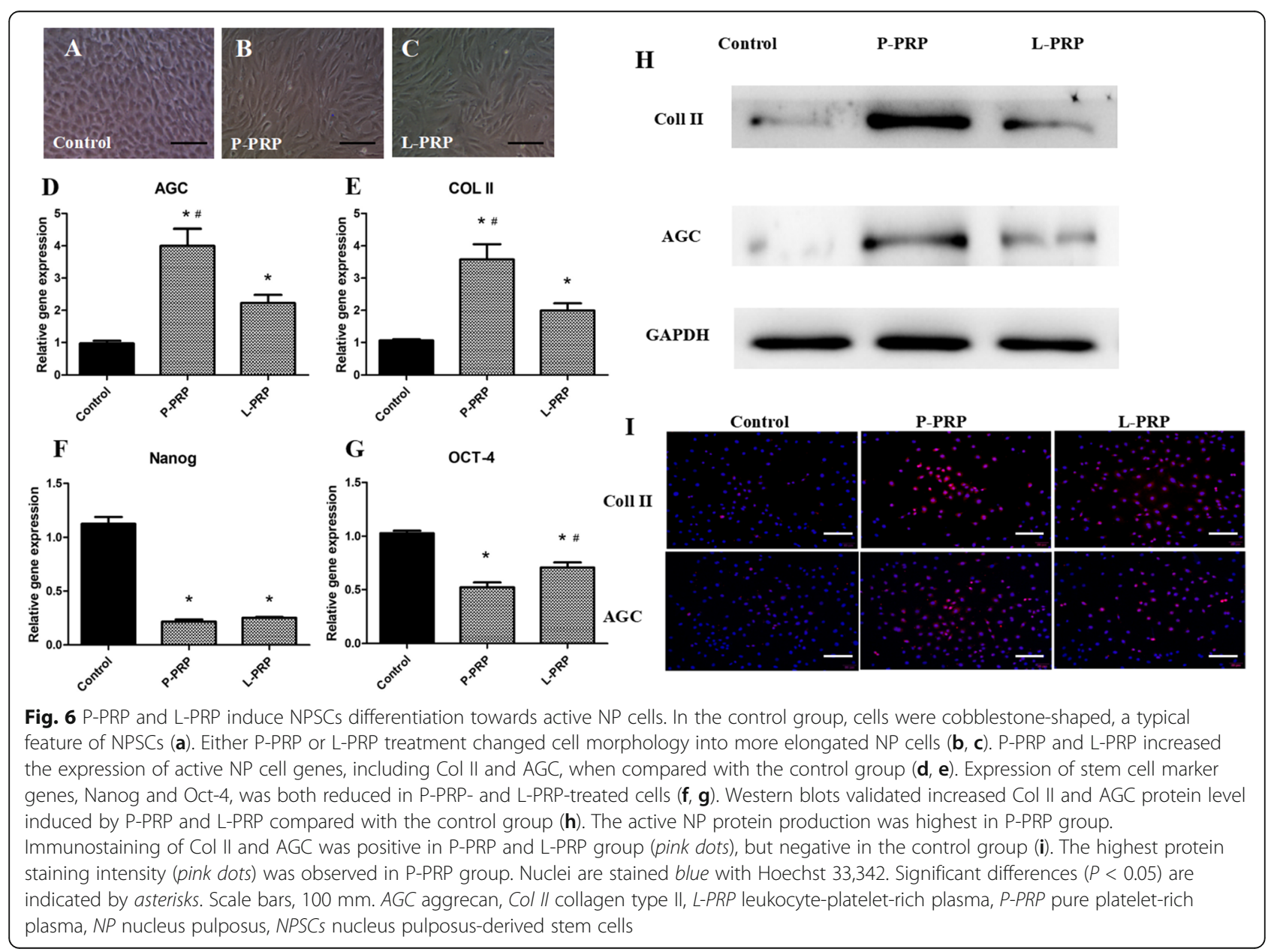



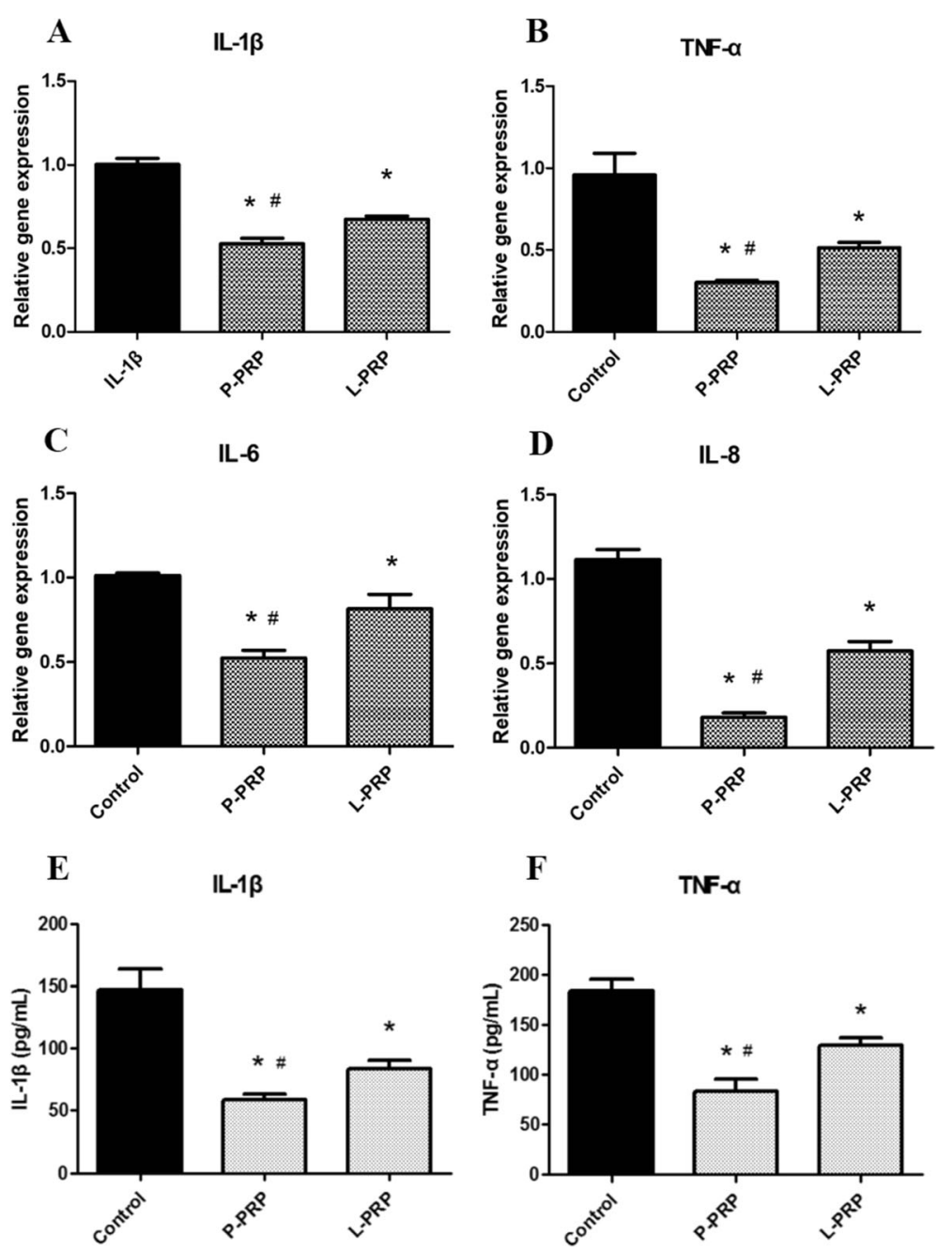

Fig. 7 P-PRP induces less inflammatory responses than L-PRP. P-PRP and L-PRP both decreased the inflammatory gene expression of IL-1 $\beta$ and TNF-a (a-d). The inflammatory gene expression was significantly lower in P-PRP group. Moreover, the level of inflammatory cytokines (IL-1 $\beta$, TNFa) in the cell supernatants was consistent with the above gene expression data (e-f). Asterisks represent significant differences when compared with the respective control, and the pound symbols indicate significant differences between L-PRP and P-PRP $(P<0.05)$. IL interleukin, L-PRP leukocyte-platelet-rich plasma, P-PRP pure platelet-rich plasma, TNF tumor necrosis factor

self-restorer when activated by P-PRP. In the early stage of IDD, the endogenic stem cells still existed, but the bioactivity was impaired. Compared to the colony-forming cells isolated from the healthy NP tissues, these cells from the early degenerated NP tissues formed less colonies and proliferated slower. A recent study showed similar results when comparing the stem cells isolated from human degenerated NP tissues with those from healthy ones [40]. The T2 signal intensity of MRI showed the worsening degeneration of the punctured discs with the extension of time in this study. Thus, active intervention for IDD in the early stage is particularly important for intervertebral disc regeneration.

To determine whether the isolated colony-forming cells expressed typical surface antigens of MSCs, we did not test the classic surface markers of MSCs for the lack of specific rabbit antigens by flow cytometry. Therefore, we used PCR to confirm the expression of these markers from gene level. The NP-derived colony-forming cells isolated from early degenerated discs (D-NPSCs) and healthy discs (H-NPSCs) both had strong expression of MSC-related markers (CD29, CD44, and CD166). Meanwhile, they had negligible expression of hematopoietic markers (CD4, CD8, and CD14), which seldom exist in MSCs. As controls, rabbit spleen cells, mostly lymphocytes and monocytes, were found to be strongly positive for both hematopoietic and MSC markers, while NP cells exhibited negligible expression of all the above genes.

The results of this study showed that the optimal proliferation rate of induced NPSCs was obtained at 10\% P-PRP concentration. A dose-dependent effect was observed below 10\% P-PRP concentration, which could be explained by more growth factors released from increasing PRP 

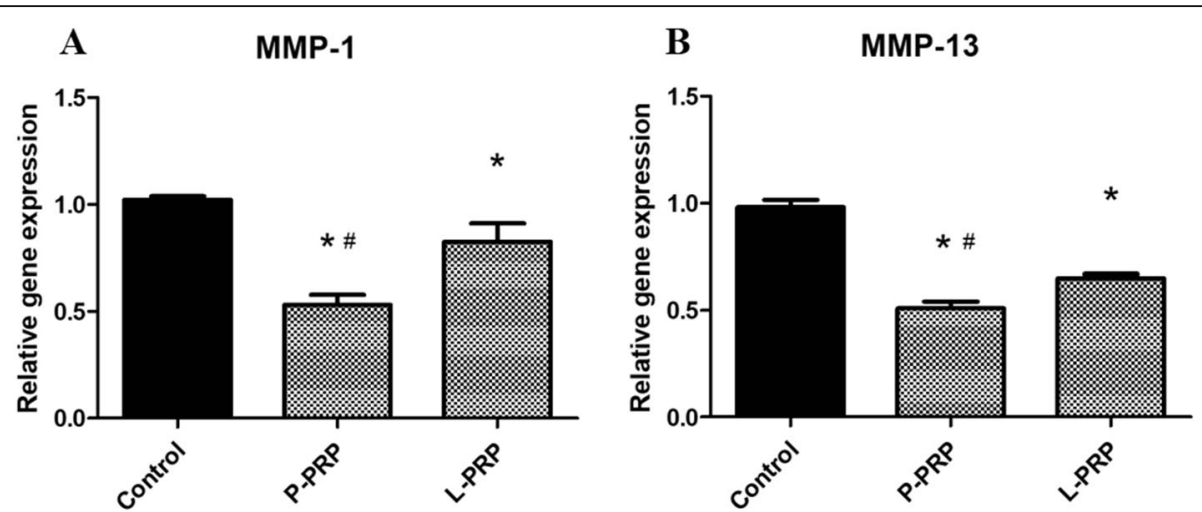

Fig. 8 P-PRP downregulates more extensive catabolic responses. P-PRP and L-PRP both significantly downregulated the gene expression of MMPs (MMP-1, MMP-13) when compared with the control (a, b). P-PRP significantly downregulated the above catabolic genes compared with L-PRP. Asterisks represent significant differences when compared with the respective control, and the pound symbols indicate significant differences between L-PRP and P-PRP $(P<0.05)$. L-PRP leukocyte-platelet-rich plasma, MMP matrix metalloproteinase, P-PRP pure platelet-rich plasma

concentration. However, excessive supplementation of P-PRP in this study resulted in decreased proliferation rate, which was also confirmed in a recent study [25]. Thus, when designed in clinical use, PRP concentration and leukocyte exclusion should be carefully tested to achieve the optimal outcome.

Previous studies have not specifically investigated the differential effects of L-PRP and P-PRP on NPSCs. Our findings demonstrated that both P-PRP and L-PRP could induce differentiation of NPSCs into active NP cells. Analysis of stem cell markers, including OCT-4 and Nanog, indicated that both P-PRP and L-PRP decreased the stemness of NPSCs in vitro. The gene expression and protein production of NP-related ECM, including collagen II and aggrecan, was significantly upregulated. However, we noticed that P-PRP was superior in collagen II and aggrecan gene expression compared to L-PRP. Thus, when treating the early IDD, P-PRP injection may promote more synthesis and accumulation of NP-related ECM, which is important in the mechanical and biological reconstruction of the degenerated intervertebral discs.

Compared to the control group, P-PRP and L-PRP both decreased the inflammation and catabolic gene expression level (IL-1 $\beta$, TNF- $\alpha$, IL-6, IL-8, MMP-, and MMP-13) of the induced NPSCs. The results were similar to a study confirming that PRP was able to suppress cytokine-induced pro-inflammatory degrading enzymes and mediators in NP cells [41]. The inflammation and catabolic gene expression levels were significantly lower in P-PRP. These findings suggest that P-PRP may exert a lower inflammation influence on the treated degenerated intervertebral discs. Therefore, the preparation of PRP is a critical factor in IDD treatment, because different leukocyte levels can contribute to variances in therapeutic efficacy. A commonly used two-stage centrifugation method was applied in many commercial kits for clinical use. It was reported that PRP prepared by two commercial kits (GPS and ACP) contained active forms of MMPs, leading to the impairment of tissue regeneration [18]. Thus, a more advanced kit should be further designed to exclude leukocytes when applied in clinical treatment.

In this study, we did not investigate the effects of P-PRP on healthy NPSCs, but rather NPSCs from early degenerated discs. As suggested in IDD therapy based on biological strategies, early intervention is the key point for regeneration $[9,42,43]$. Previous studies have confirmed that the proliferation and differentiation potentials of NPSCs are worsening in the degeneration process $[24,40,44]$. In this study, we noticed that P-PRP and L-PRP both induced NPSCs from early degenerated discs into active NP cells by upregulating collagen II and aggrecan gene expression and protein synthesis. The results were consistent with some in vivo studies, which proved that PRP injection was helpful in restoring the ECM content, thus prohibiting the degeneration process $[28,45,46]$. However, we did not test the efficacy of P-PRP on the NPSCs from advanced degenerated intervertebral discs for those three reasons. First, in the late stage of IDD, NP structure collapses and can hardly be collected from rabbit models used in this study. Second, IDD is a progressive, chronic disease; over time, the degeneration worsens and ultimately becomes irreversible; in the late stage of IDD, the calcified endplate holds limited potential for vascularization and nutrient delivery for the starving cells $[47,48]$. Third, IDD reconstruction by tissue engineering holds promise for late stage of IDD $[49,50]$; the use of PRP is more beneficial when co-applied with more active seeding cells, but not the cells in degeneration or even senescence.

PRP has become a promising agent for treating surgical site infections for the antibacterial activity [21]. However, as for IDD, which mostly is related to the chronic low back pain, excluding leukocytes in PRP would be a 
more effective choice. The presence of pro-inflammatory cytokines and mediators is characterized as the prominent change in IDD [51]. The decreased inflammatory cytokines can prohibit the unwanted inflammatory-related pain and unnecessary ECM degradation. The injection agent for IDD should be safe, together with low pro-inflammatory effects. Leukocytes in PRP may only exacerbate IDD by prolonging the inflammatory process, leading to worsening pain experience in patients. Thus, P-PRP exhibits a superior choice compared to L-PRP in IDD treatment.

\section{Conclusions}

In this study, we confirmed that the colony-forming cells (NPSCs) could be isolated from the NP tissues of early degenerated intervertebral discs. PRP, including P-PRP and L-PRP, could proliferate NPSCs and activate these cells toward active NP cells. Both two types of PRP could suppress the inflammatory levels of NPSCs cultured in vitro. However, P-PRP exerted a significantly lower catabolic and inflammatory effect on NPSCs. These results explain the exclusion of leukocytes from PRP may be a more effective choice for IDD in early stage.

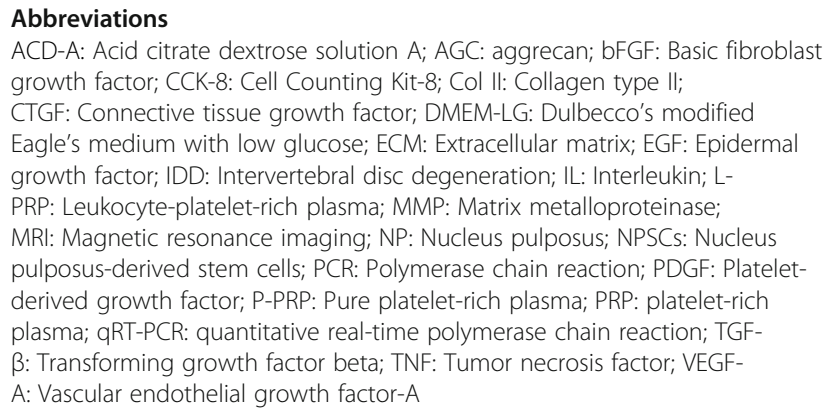
growth factor; CCK-8: Cell Counting Kit-8; Col II: Collagen type II; CTGF: Connective tissue growth factor; DMEM-LG: Dulbecco's modified Eagle's medium with low glucose; ECM: Extracellular matrix; EGF: Epidermal growth factor; IDD: Intervertebral disc degeneration; IL: Interleukin; LPRP: Leukocyte-platelet-rich plasma; MMP: Matrix metalloproteinase; MRI: Magnetic resonance imaging; NP: Nucleus pulposus; NPSCs: Nucleus pulposus-derived stem cells; PCR: Polymerase chain reaction; PDGF: Plateletderived growth factor; P-PRP: Pure platelet-rich plasma; PRP: platelet-rich plasma; qRT-PCR: quantitative real-time polymerase chain reaction; TGF$\beta$ : Transforming growth factor beta; TNF: Tumor necrosis factor; VEGFA: Vascular endothelial growth factor-A

\section{Acknowledgements}

The funding support from the National Natural Science Foundation of China (Grant No: 81572188) for this work is gratefully acknowledged (CW).

\section{Funding}

Already stated above.

\section{Availability of data and materials}

Please contact author for data requests.

\section{Authors' contributions}

SZW performed the experiments, collected data, and drafted the manuscript. $\mathrm{JJ}$ participated in the experimental design and experimentation, and helped collect the data. LYM and JBY participated in designing this study and interpreting the experimental results. CW took part in the study design, provided clinical perspectives for the findings of this study, and revised the manuscript. WMF conceived the study, designed the experiments, interpreted the data, and drafted the manuscript. All authors read and approved the final manuscript.

\section{Ethics approval}

The Institutional Animal Care and Use Committee of Southeast University approved the protocol for the use of rabbits in this study.

\section{Consent for publication}

Not applicable.

\section{Competing interests}

The authors declare that they have no competing interests.

\section{Publisher's Note}

Springer Nature remains neutral with regard to jurisdictional claims in published maps and institutional affiliations.

Received: 16 February 2018 Revised: 7 June 2018 Accepted: 19 June 2018 Published online: 18 July 2018

\section{References}

1. Freemont AJ, Watkins A, Le MC, Jeziorska M, Hoyland JA. Current understanding of cellular and molecular events in intervertebral disc degeneration: implications for therapy. J Pathol. 2002;196(4):374-9.

2. Freemont AJ. The cellular pathobiology of the degenerate intervertebral disc and discogenic back pain. Rheumatology (Oxford). 2009;48(1):5-10.

3. Ren C, Li Y, Qin R, Sun P, Wang P. Transforaminal endoscopic lumbar discectomy for lumbar disc herniation causing bilateral symptoms. World Neurosurg. 2017;106:413-21.

4. Sairyo K, Chikawa T, Nagamachi A. State-of-the-art transforaminal percutaneous endoscopic lumbar surgery under local anesthesia: discectomy, foraminoplasty, and ventral facetectomy. J Orthop Sci. 2017;

5. Karppinen J, Shen FH, Luk KD, Andersson GB, Cheung KM, Samartzis D. Management of degenerative disk disease and chronic low back pain. Orthop Clin North Am. 2011;42(4):513-28. viii

6. Wang $S Z$, Rui YF, Tan Q, Wang C. Enhancing intervertebral disc repair and regeneration through biology: platelet-rich plasma as an alternative strategy. Arthritis Res Ther. 2013;15(5):220.

7. Akeda K, Ohishi K, Masuda K, et al. Intradiscal injection of autologous platelet-rich plasma Releasate to treat Discogenic low back pain: a preliminary clinical trial. Asian Spine J. 2017;11(3):380-9.

8. Levi D, Horn S, Tyszko S, Levin J, Hecht-Leavitt C, Walko E. Intradiscal platelet-rich plasma injection for chronic Discogenic low back pain: preliminary results from a prospective trial. Pain Med. 2016;17(6):1010-22.

9. Wang SZ, Chang Q, Lu J, Wang C. Growth factors and platelet-rich plasma: promising biological strategies for early intervertebral disc degeneration. Int Orthop. 2015;39(5):927-34.

10. Marx RE. Platelet-rich plasma (PRP): what is PRP and what is not PRP. Implant Dent. 2001;10(4):225-8.

11. Follo F, Dejana DO, Belletti M, et al. Management and effect of platelet-rich plasma on wound healing: small reality of Oglio Po hospital. Acta Biomed. 2017;88(5-S):66-70.

12. Borrione P, Fagnani F, Di GA, Mancini A, Pigozzi F, Pitsiladis Y. The role of platelet-rich plasma in muscle healing. Curr Sports Med Rep. 2017; 16(6):459-63.

13. Reinders $\mathrm{Y}$, Felthaus $\mathrm{O}$, Brockhoff $\mathrm{G}$, et al. Impact of platelet-rich plasma on viability and proliferation in wound healing processes after external radiation. Int J Mol Sci. 2017;18(8)

14. Alzahrani AA, Murriky A, Shafik S. Influence of platelet rich fibrin on postextraction socket healing: a clinical and radiographic study. Saudi Dent J. 2017;29(4):149-55

15. Leslie M. Cell biology. Beyond clotting: the powers of platelets. Science. 2010;328(5978):562-4.

16. Sánchez-González DJ, Méndez-Bolaina E, Trejo-Bahena NI. Platelet-rich plasma peptides: key for regeneration. Int J Pept. 2012;2012:532519.

17. Chahla J, Cinque ME, Piuzzi NS, et al. A call for standardization in plateletrich plasma preparation protocols and composition reporting: a systematic review of the clinical Orthopaedic literature. J Bone Joint Surg Am. 2017; 99(20):1769-79.

18. Pifer MA, Maerz T, Baker KC, Anderson K. Matrix metalloproteinase content and activity in low-platelet, low-leukocyte and high-platelet, high-leukocyte platelet rich plasma (PRP) and the biologic response to PRP by human ligament fibroblasts. Am J Sports Med. 2014;42(5):1211-8.

19. Kobayashi Y, Saita Y, Nishio H, et al. Leukocyte concentration and composition in platelet-rich plasma (PRP) influences the growth factor and protease concentrations. J Orthop Sci. 2016;21(5):683-9.

20. López-Cotarelo P, Gómez-Moreira C, Criado-García O, Sánchez L, RodríguezFernández JL. Beyond Chemoattraction: multifunctionality of chemokine receptors in leukocytes. Trends Immunol. 2017;38(12):927-41. 
21. Cetinkaya RA, Yilmaz S, Ünlü A, et al. The efficacy of platelet-rich plasma gel in MRSA-related surgical wound infection treatment: an experimental study in an animal model. Eur J Trauma Emerg Surg. 2017;

22. Hadjipavlou AG, Tzermiadianos MN, Bogduk N, Zindrick MR. The pathophysiology of disc degeneration: a critical review. J Bone Joint Surg Br. 2008:90(10):1261-70.

23. Sakai D, Nakamura Y, Nakai T, et al. Exhaustion of nucleus pulposus progenitor cells with ageing and degeneration of the intervertebral disc. Nat Commun. 2012;3:1264.

24. Wu H, Zeng X, Yu J, et al. Comparison of nucleus pulposus stem/progenitor cells isolated from degenerated intervertebral discs with umbilical cord derived mesenchymal stem cells. Exp Cell Res. 2017;361(2):324-32.

25. Zhou Y, Zhang J, Wu H, Hogan MV, Wang JH. The differential effects of leukocyte-containing and pure platelet-rich plasma (PRP) on tendon stem/ progenitor cells - implications of PRP application for the clinical treatment of tendon injuries. Stem Cell Res Ther. 2015;6:173.

26. Yin W, Qi X, Zhang Y, et al. Advantages of pure platelet-rich plasma compared with leukocyte- and platelet-rich plasma in promoting repair of bone defects. J Transl Med. 2016;14:73.

27. Yin WJ, Xu HT, Sheng JG, et al. Advantages of pure platelet-rich plasma compared with leukocyte- and platelet-rich plasma in treating rabbit knee osteoarthritis. Med Sci Monit. 2016;22:1280-90.

28. Obata S, Akeda K, Imanishi T, et al. Effect of autologous platelet-rich plasmareleasate on intervertebral disc degeneration in the rabbit anular puncture model: a preclinical study. Arthritis Res Ther. 2012;14(6):R241.

29. Lee TC, Lee TH, Huang YH, Chang NK, Lin YJ, Chien PW, Yang WH, Lin MH. Comparison of surface markers between human and rabbit mesenchymal stem cells. PLoS One. 2014;9:e111390. https://doi.org/10.1371/journal.pone.0111390.

30. Zhang $X, \mathrm{Li}$ J, Nie J, et al. Differentiation character of adult mesenchymal stem cells and transfection of MSCs with lentiviral vectors. J Huazhong Univ Sci Technolog Med Sci. 2010;30(6):687-93.

31. Huang $H$, Zhang $X, H u X$, et al. Directing chondrogenic differentiation of mesenchymal stem cells with a solid-supported chitosan thermogel for cartilage tissue engineering. Biomed Mater. 2014;9(3):035008.

32. Kon E, Filardo G, Di MA, Marcacci M. Platelet-rich plasma (PRP) to treat sports injuries: evidence to support its use. Knee Surg Sports Traumatol Arthrosc. 2011;19(4):516-27.

33. Valentí AA, Lamo-Espinosa J, Aquerreta BJD, Hernandez GM, Mora GG, Valentí NJR. Comparison between two different platelet-rich plasma preparations and control applied during anterior cruciate ligament reconstruction. Is there any evidence to support their use. Injury. 2014; 45(Suppl 4):S36-41.

34. Bastami $F$, Vares $P$, Khojasteh A. Healing effects of platelet-rich plasma on peripheral nerve injuries. J Craniofac Surg. 2017;28(1):e49-57.

35. Fernandes $\mathrm{G}$, Yang S. Application of platelet-rich plasma with stem cells in bone and periodontal tissue engineering. Bone Res. 2016;4:16036.

36. Mlynarek RA, Kuhn AW, Bedi A. Platelet-rich plasma (PRP) in orthopedic sports medicine. Am J Orthop (Belle Mead NJ). 2016;45(5):290-326.

37. Formica M, Cavagnaro L, Formica C, Mastrogiacomo M, Basso M, Di MA. What is the preclinical evidence on platelet rich plasma and intervertebral disc degeneration. Eur Spine J. 2015;24(11):2377-86.

38. Monfett M, Harrison J, Boachie-Adjei K, Lutz G. Intradiscal platelet-rich plasma (PRP) injections for discogenic low back pain: an update. Int Orthop. 2016;40(6):1321-8.

39. Pavlovic V, Ciric M, Jovanovic V, Stojanovic P. Platelet rich plasma: a short overview of certain bioactive components. Open Med (Wars). 2016;11(1):242-7.

40. Jia Z, Yang $P, W u Y$, et al. Comparison of biological characteristics of nucleus pulposus mesenchymal stem cells derived from nondegenerative and degenerative human nucleus pulposus. Exp Ther Med. 2017;13(6):3574-80

41. Kim HJ, Yeom JS, Koh YG, et al. Anti-inflammatory effect of platelet-rich plasma on nucleus pulposus cells with response of TNF-a and IL-1. J Orthop Res. 2014;32(4):551-6.

42. Longo UG, Petrillo S, Franceschetti E, Maffulli N, Denaro V. Growth factors and anticatabolic substances for prevention and management of intervertebral disc degeneration. Stem Cells Int. 2012;2012:897183.

43. Masuda K, An HS. Prevention of disc degeneration with growth factors. Eur. Spine J. 2006;15(Suppl 3):S422-32

44. LV F, Leung VY, Huang S, Huang Y, Sun Y, Cheung KM. In search of nucleus pulposus-specific molecular markers. Rheumatology (Oxford). 2014;53(4): 600-10.
45. Sawamura K, Ikeda T, Nagae M, et al. Characterization of in vivo effects of platelet-rich plasma and biodegradable gelatin hydrogel microspheres on degenerated intervertebral discs. Tissue Eng Part A. 2009;15(12):3719-27.

46. Nagae $M$, Ikeda $T$, Mikami $Y$, et al. Intervertebral disc regeneration using platelet-rich plasma and biodegradable gelatin hydrogel microspheres. Tissue Eng. 2007;13(1):147-58.

47. Chan WC, Sze KL, Samartzis D, Leung WY, Chan D. Structure and biology of the intervertebral disk in health and disease. Orthop Clin North Am. 2011; 42(4):447-64. vii

48. Sakai D, Grad S. Advancing the cellular and molecular therapy for intervertebral disc disease. Adv Drug Deliv Rev. 2015:84:159-71.

49. Vadalà G, Russo F, Di MA, Denaro V. Intervertebral disc regeneration: from the degenerative cascade to molecular therapy and tissue engineering. J Tissue Eng Regen Med. 2015;9(6):679-90.

50. van Uden S, Silva-Correia J, Oliveira JM, Reis RL. Current strategies for treatment of intervertebral disc degeneration: substitution and regeneration possibilities. Biomater Res. 2017;21:22.

51. Li W, Liu T, Wu L, et al. Blocking the function of inflammatory cytokines and mediators by using IL-10 and TGF- $\beta$ : a potential biological immunotherapy for intervertebral disc degeneration in a beagle model. Int J Mol Sci. 2014; 15(10):17270-83.

\section{Ready to submit your research? Choose BMC and benefit from:}

- fast, convenient online submission

- thorough peer review by experienced researchers in your field

- rapid publication on acceptance

- support for research data, including large and complex data types

- gold Open Access which fosters wider collaboration and increased citations

- maximum visibility for your research: over $100 \mathrm{M}$ website views per year

At BMC, research is always in progress.

Learn more biomedcentral.com/submissions 